\title{
Spinal anesthesia in awake surgical procedures of the lumbar spine: a systematic review and meta-analysis of 3709 patients
}

\author{
Roberto J. Perez-Roman, MD, Vaidya Govindarajan, BS, Jean-Paul Bryant, MS, and \\ Michael Y. Wang, MD \\ Department of Neurological Surgery and The Miami Project to Cure Paralysis, University of Miami Miller School of Medicine, \\ Miami, Florida
}

\begin{abstract}
OBJECTIVE Awake surgery has previously been found to improve patient outcomes postoperatively in a variety of procedures. Recently, multiple groups have investigated the utility of this modality for use in spine surgery. However, few current meta-analyses exist comparing patient outcomes in awake spinal anesthesia with those in general anesthesia. Therefore, the authors sought to present an updated systematic review and meta-analysis investigating the utility of spinal anesthesia relative to general anesthesia in lumbar procedures.
\end{abstract}

METHODS Following a comprehensive literature search of the PubMed and Cochrane databases, 14 clinical studies were included in our final qualitative and quantitative analyses. Of these studies, 5 investigated spinal anesthesia in lumbar discectomy, 4 discussed lumbar laminectomy, and 2 examined interbody fusion procedures. One study investigated combined lumbar decompression and fusion or decompression alone. Two studies investigated patients who underwent discectomy and laminectomy, and 1 study investigated a series of patients who underwent transforaminal lumbar interbody fusion, posterolateral fusion, or decompression. Odds ratios, mean differences (MDs), and 95\% confidence intervals were calculated where appropriate.

RESULTS A meta-analysis of the total anesthesia time showed that time was significantly less in patients who received spinal anesthesia for both lumbar discectomies (MD $-26.53,95 \% \mathrm{Cl}-38.16$ to $-14.89 ; p=0.00001$ ) and lumbar laminectomies (MD $-11.21,95 \% \mathrm{Cl}-19.66$ to $-2.75 ; p=0.009$ ). Additionally, the operative time was significantly shorter in patients who underwent spinal anesthesia (MD $-14.94,95 \% \mathrm{Cl}-20.43$ to $-9.45 ; p<0.00001)$. Similarly, when analyzing overall postoperative complication rates, patients who received spinal anesthesia were significantly less likely to experience postoperative complications (OR $0.29,95 \% \mathrm{Cl} 0.16-0.53 ; \mathrm{p}<0.0001)$. Furthermore, patients who received spinal anesthesia had significantly lower postoperative pain scores (MD $-2.80,95 \% \mathrm{Cl}-4.55$ to $-1.06 ; p=0.002)$. An identical trend was seen when patients were stratified by lumbar procedures. Patients who received spinal anesthesia were significantly less likely to require postoperative analgesia (OR $0.06,95 \% \mathrm{Cl} 0.02-0.25 ; p<0.0001$ ) and had a significantly shorter hospital length of stay (MD $-0.16,95 \% \mathrm{Cl}-0.29$ to $-0.03 ; p=0.02$ ) and intraoperative blood loss (MD -52.36 , $95 \% \mathrm{Cl}-81.55$ to $-23.17 ; p=0.0004)$. Finally, the analysis showed that spinal anesthesia cost significantly less than general anesthesia (MD $-226.14,95 \% \mathrm{Cl}-324.73$ to $-127.55 ; p<0.00001)$.

CONCLUSIONS This review has demonstrated the varying benefits of spinal anesthesia in awake spine surgery relative to general anesthesia in patients who underwent various lumbar procedures. The analysis has shown that spinal anesthesia may offer some benefits when compared with general anesthesia, including reduction in the duration of anesthesia, operative time, total cost, and postoperative complications. Large prospective trials will elucidate the true role of this modality in spine surgery.

https://thejns.org/doi/abs/10.3171/2021.9.FOCUS21464

KEYWORDS spinal anesthesia; lumbar; operative time; complications; awake surgery

ABBREVIATIONS LOS = length of stay; MD = mean difference; VAS = visual analog scale

SUBMITTED August 1, 2021. ACCEPTED September 23, 2021.

INCLUDE WHEN CITING DOI: 10.3171/2021.9.FOCUS21464. 
$\mathrm{A}$ WAKE surgery has been utilized as a primary surgical technique across multiple disciplines. In neurosurgery, awake procedures are a mainstay in certain cranial approaches that require resections near eloquent areas. ${ }^{1}$ However, general anesthesia has traditionally been the primary means of sedation in spine procedures, with awake modalities largely considered a secondary option in patients with multiple comorbidities or in elderly patients. This order of selection has endured despite the well-documented disadvantages associated with the use of general anesthetics in certain patient populations, including longer operative time, postoperative delirium, and a potential increase in pain medication requirement. ${ }^{2}$ Furthermore, recent studies have documented multiple benefits when replacing general anesthesia with awake procedures, most notably, increased patient satisfaction and reduced hospital length of stay (LOS). ${ }^{3}$

Increased use of minimally invasive procedures in treatment of spinal pathologies has sparked increased interest in the utility of awake spinal anesthesia techniques because awake techniques allow surgeons to attain instant patient feedback when assessing the proximity of surgical instruments to neural elements. ${ }^{4}$ However, while multiple researchers have documented the results of studies investigating awake spinal anesthesia techniques, few systematic reviews or meta-analyses currently exist that compare perioperative outcomes between spinal anesthesia administered to patients undergoing awake surgery and general anesthesia. Meng et al. concluded, in a systematic review, that spinal anesthesia was associated with decreased intraoperative hypertension, tachycardia, and postoperative analgesia requirements, but they failed to report some important surgical variables, including anesthesia time and postoperative complications. ${ }^{5}$ Furthermore, current systematic reviews and meta-analyses do not offer a comprehensive examination of available intraoperative and perioperative metrics in addition to cost analysis. ${ }^{5-7}$ Given the increased volume of spine procedures due to the aging population and initiatives to deliver accessible and affordable healthcare to all patients, a comprehensive systematic review evaluating spinal anesthesia would be of clinical value, particularly with respect to patient selection and postoperative outcomes. Here, we present a comprehensive systematic review and meta-analysis investigating the potential advantages of using spinal anesthesia compared with general anesthesia in lumbar surgical procedures.

\section{Methods}

\section{Inclusion and Exclusion Criteria}

Studies comparing awake spinal surgery with spinal surgery under general anesthesia and their clinical outcomes in patients who underwent lumbar surgery were included. The exclusion criteria were 1) nonhuman studies, 2) cohorts that did not incorporate surgical management, 3) studies that did not specifically compare spinal anesthesia with general anesthesia, 4) cohorts that did not evaluate clinical outcomes, and 5) case reports.

\section{Literature Search and Selection}

A systematic search of English-language literature from July 1990 to July 2021 in the PubMed and Cochrane databases was performed. The following medical subject headings (MeSH) and free-text words were used: "spinal neurosurgery" or "spinal neurological surgery" and "awake" and ("anesthesia" or "local anesthesia") "spinal anesthesia" and ("percutaneous" or "endoscopic") and "spine," "spinal anesthesia" and "lumbar" and "spine" and "surgery," and "awake spine surgery." A total of 552 published abstracts or articles were identified. After screening and application of strict inclusion and exclusion criteria, a total of 14 articles were included in the qualitative analysis and 14 in the quantitative analysis (Fig. 1). Selection and extraction of the studies was performed by two independent authors (V.G. and J.P.B.).

\section{Data Analysis}

The pooled data analysis was performed using Review Manager version 5.4 (RevMan; The Cochrane Collaboration). Odds ratios, mean differences (MDs), and 95\% confidence intervals were calculated using the MantelHaenszel test, and forest plots were generated for each comparison. Random-effects dichotomous and continuous models were developed to assess the overall effect of each anesthetic in the control and treatment groups. Primary outcomes were anesthesia duration, operative time, and complication rates. Complications included infection, dural injury, postoperative hematomas, urinary retention, cardiopulmonary events, postoperative nausea/vomiting, or hemodynamic instability. Secondary outcomes included postoperative pain represented by the visual analog scale (VAS), intraoperative blood loss, postoperative analgesia use, and anesthesia cost. The risk of bias was assessed using the Cochrane risk-of-bias tool in order to provide further insight into the conclusions made by the authors and our own subsequent meta-analyses (Fig. 2).

\section{Results}

\section{Study Selection and Characteristics}

Overall, 14 articles met the inclusion and exclusion criteria from an initial pool of 552 articles. ${ }^{8-21}$ All articles evaluated the total intraoperative time, anesthesia duration, postoperative complications, postoperative pain, or a combination thereof. All 14 articles were included in the quantitative analysis and compared the aforementioned outcomes in patients who received general anesthesia with those who received spinal anesthesia and underwent an awake procedure. Furthermore, all included articles reported performing lumbar spine surgeries. Key differences in the articles are summarized in Table 1. Our riskof-bias summary is presented in Fig. 2. An overview of the anesthesia protocols used in the articles included for final review is displayed in Table 1.

\section{Outcomes in Lumbar Discectomy}

Of 14 articles, 5 evaluated the use of spinal anesthesia in patients who underwent lumbar discectomy. ${ }^{9-11,14,19}$ In all studies, the total operative time was reported in minutes. Our pooled analysis demonstrated that among patients who underwent lumbar discectomy, patients who received spinal anesthesia had significantly shorter mean operating 


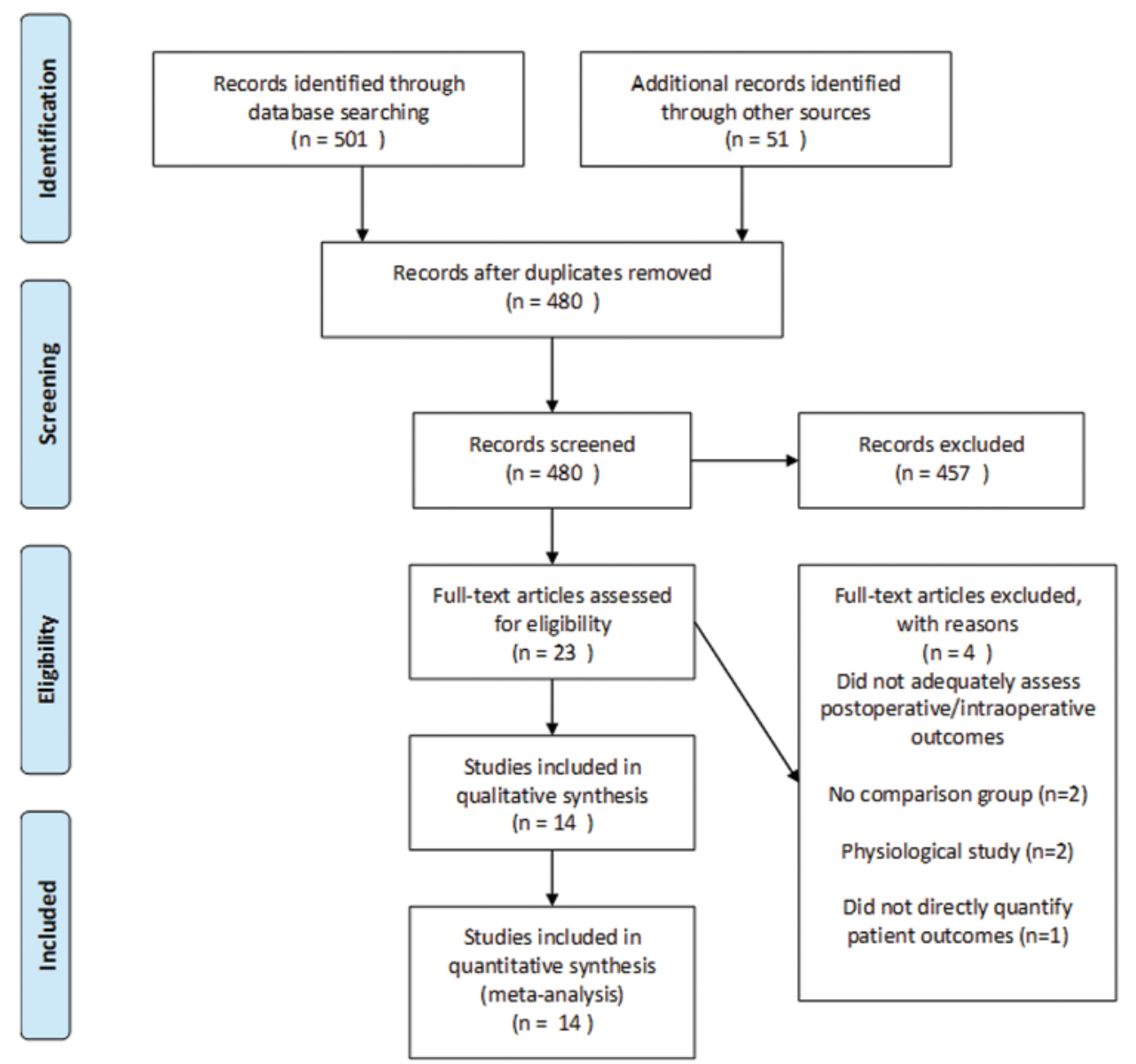

FIG. 1. PRISMA flow diagram of inclusion and exclusion criteria. Data added to the PRISMA template [from Moher D, Liberati A, Tetzlaff J, Altman DG, The PRISMA Group (2009). Preferred Reporting Items for Systematic Reviews and Meta-Analyses: The PRISMA Statement. PLoS Med 6(7):e1000097 under the terms of the Creative Commons Attribution License.

times relative to patients who received general anesthesia (MD $-14.04,95 \%$ CI -21.30 to $-6.79 ; \mathrm{p}=0.0001)$ (Fig. 3A). Similarly, patients who underwent spinal anesthesia had a significantly shorter total anesthesia time (MD $-26.53,95 \%$ CI -38.16 to $-14.89 ; \mathrm{p}=0.00001$ ) (Fig. 3B). Furthermore, patients who received spinal anesthesia were significantly less likely to experience postoperative complications than patients who received general anesthesia (OR 0.18, 95\% CI 0.09-0.35; p < 0.00001) (Fig. 3C). Our third pooled analysis demonstrated that patients who underwent spinal anesthesia reported lower VAS pain scores postoperatively than patients who underwent general anesthesia (MD $-2.50,95 \%$ CI -3.91 to $-1.09 ; \mathrm{p}=0.0005$ ) (Fig. 3D). Finally, there were no significant differences in LOS between patients who received spinal anesthesia and those who received general anesthesia (MD $-0.10,95 \%$ CI -0.25 to $0.06 ; p=0.22$ ) (Fig. 3E).

\section{Outcomes in Lumbar Laminectomy}

Four articles evaluated the use of spinal anesthesia in patients who underwent lumbar laminectomy for either spinal stenosis or herniated discs. ${ }^{13-15,20}$ A pooled analysis demonstrated that there were significant differences in total operative time between patients who received spi- nal anesthesia and those who received general anesthesia (MD -10.60, 95\% CI -20.64 to -0.55; $\mathrm{p}=0.04$ ) (Fig. 4A). Similarly, there were statistically significant differences in complication risk (OR 0.33, 95\% CI 0.11-0.99; $p=0.05$ ), as seen in Fig. 4B. Additionally, the total anesthesia time was significantly shorter for patients who underwent spinal anesthesia than those who received general anesthesia (MD -11.21, 95\% CI -19.66 to $-2.75 ; p=0.009)$ (Fig. 4C).

\section{Outcomes in Lumbar Fusion}

Two articles evaluated spinal anesthesia in patients who underwent lumbar interbody fusion. ${ }^{17,18}$ Our pooled analysis demonstrated that patients who received spinal anesthesia had significantly shorter operative times (MD $-21.51,95 \%$ CI -41.77 to -1.26 ; $p=0.04$ ) (Fig. 5 A). In addition, patients who received spinal anesthesia had significantly lower postoperative pain scores compared with those who received general anesthesia (MD -3.89, 95\% CI -6.29 to $-1.49 ; \mathrm{p}=0.001$ ) (Fig. $5 \mathrm{~B}$ ).

\section{Outcomes Across Lumbar Surgeries}

Across all studies, patients who received spinal anesthesia had significantly shorter total operative times than patients who received general anesthesia (MD -14.94, 95\% 


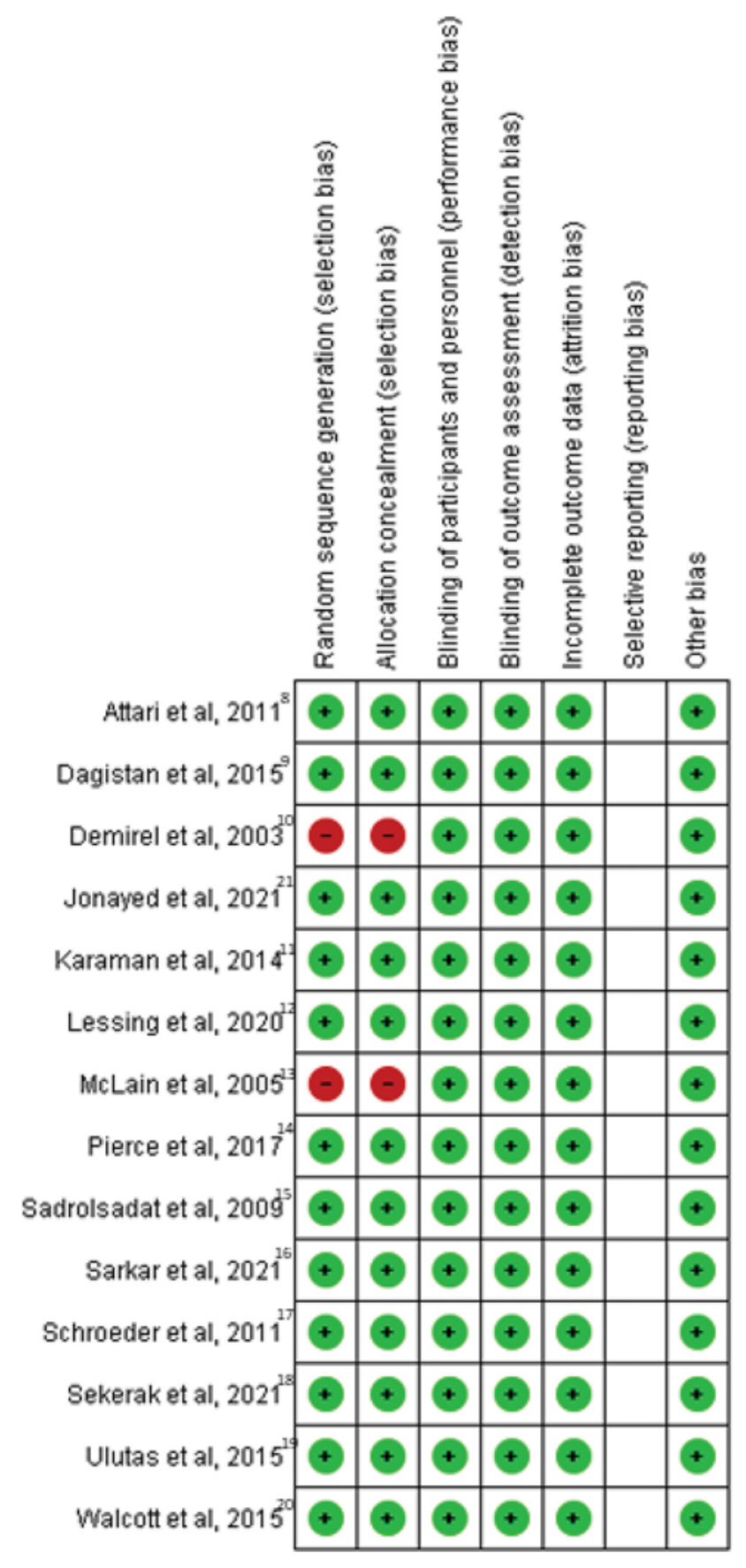

FIG. 2. Risk-of-bias summary. Green indicates low risk, red indicates risk, and a blank space indicates unknown risk.

CI -20.43 to -9.45 ; $p<0.00001$ ) (Fig. 6A). Similarly, total anesthesia times were significantly shorter in patients who received spinal anesthesia than those who received general anesthesia (MD -20.60, 95\% CI -28.18 to -13.02 ; $\mathrm{p}<0.00001$ ) (Fig. 6B). Complication rates were also significantly lower in patients who received spinal anesthesia than in patients who received general anesthesia (OR 0.29, 95\% CI 0.16-0.53; p < 0.0001) (Fig. 6C). Our pooled analysis of all studies also demonstrated that patients who received spinal anesthesia had significantly lower postop- erative VAS scores than patients who received general anesthesia (MD $-2.80,95 \% \mathrm{CI}-4.55$ to $-1.06 ; \mathrm{p}=0.002$ ) (Fig. 6D).

Furthermore, we performed pooled analyses of other clinically useful metrics across lumbar surgeries. Analysis of postoperative analgesic use revealed that patients who received spinal anesthesia were significantly less likely to require postoperative analgesia (OR $0.06,95 \%$ CI 0.02 0.25 ; $\mathrm{p}<0.0001)$ (Fig. 7A). Patients who received spinal anesthesia also had a significantly shorter mean LOS (days) than those who received general anesthesia (MD $-0.16,95 \%$ CI -0.29 to $-0.03 ; p=0.02$ ) (Fig. 7B). Spinal anesthesia was significantly less costly across all investigated studies (MD $-226.14,95 \%$ CI -324.73 to -127.55 ; $\mathrm{p}$ $<0.00001$ ) relative to general anesthesia (Fig. 7C). Patients who received spinal anesthesia also had significantly less postoperative blood loss (MD $-52.36,95 \% \mathrm{CI}-81.55$ to -23.17; $\mathrm{p}=0.0004$ ) (Fig. 7D).

\section{Discussion}

Awake surgical procedures have been previously used in a wide range of specialties; however, there is a paucity of information regarding their utility in spine surgery from a meta-analytical perspective. Thus, the current evidence in favor of spinal anesthetics remains unclear despite the growing evidence of the benefits in certain surgical patients. ${ }^{22}$ All included studies evaluated a combination of postoperative complication rates, total anesthesia time, operative time, blood loss, LOS, postoperative analgesic use, and anesthesia cost. ${ }^{8-21}$ In addition to assessing the impact of spinal anesthesia versus general anesthesia across all surgical techniques, we performed subgroup analyses separated by procedure. Our stratified analyses showed that total anesthesia times were significantly shorter in patients who received spinal anesthesia than in those who received general anesthesia. Interestingly, our pooled analyses also demonstrated that patients who received spinal anesthesia had significantly shorter total operative times compared with patients who received general anesthesia across all lumbar surgeries. In a case-control study of 400 patients, McLain et al. reported that not only did administration of spinal anesthesia decrease the total time with anesthetics but also it resulted in reduction of the total surgical time. ${ }^{13}$ This could be associated with the significant reduction of intraoperative hemodynamic events that are associated with the use of general anesthesia. ${ }^{13}$ Deng et al. identified that spinal anesthesia was associated with decreased administration of drugs and vasoactive agents intraoperatively, which may translate into greater hemodynamic stability, decreased blood loss, and a shorter operating time. ${ }^{23}$

We observed a similar trend, with respect to complication rates, with studies that performed lumbar discectomies (OR 0.18, 95\% CI $0.09-0.35 ; \mathrm{p}<0.00001$ ) and lumbar laminectomies (OR 0.33, 95\% CI 0.11-0.99; $\mathrm{p}=$ 0.05) reporting lower postoperative complication rates in patients who received spinal anesthesia. In addition, Sarkar et al. reported nearly a $20 \%$ reduction of postoperative nausea and vomiting in patients who underwent lumbar fusion. ${ }^{16}$ These findings were corroborated by Fiani et al., who reviewed the existing literature and suggested 


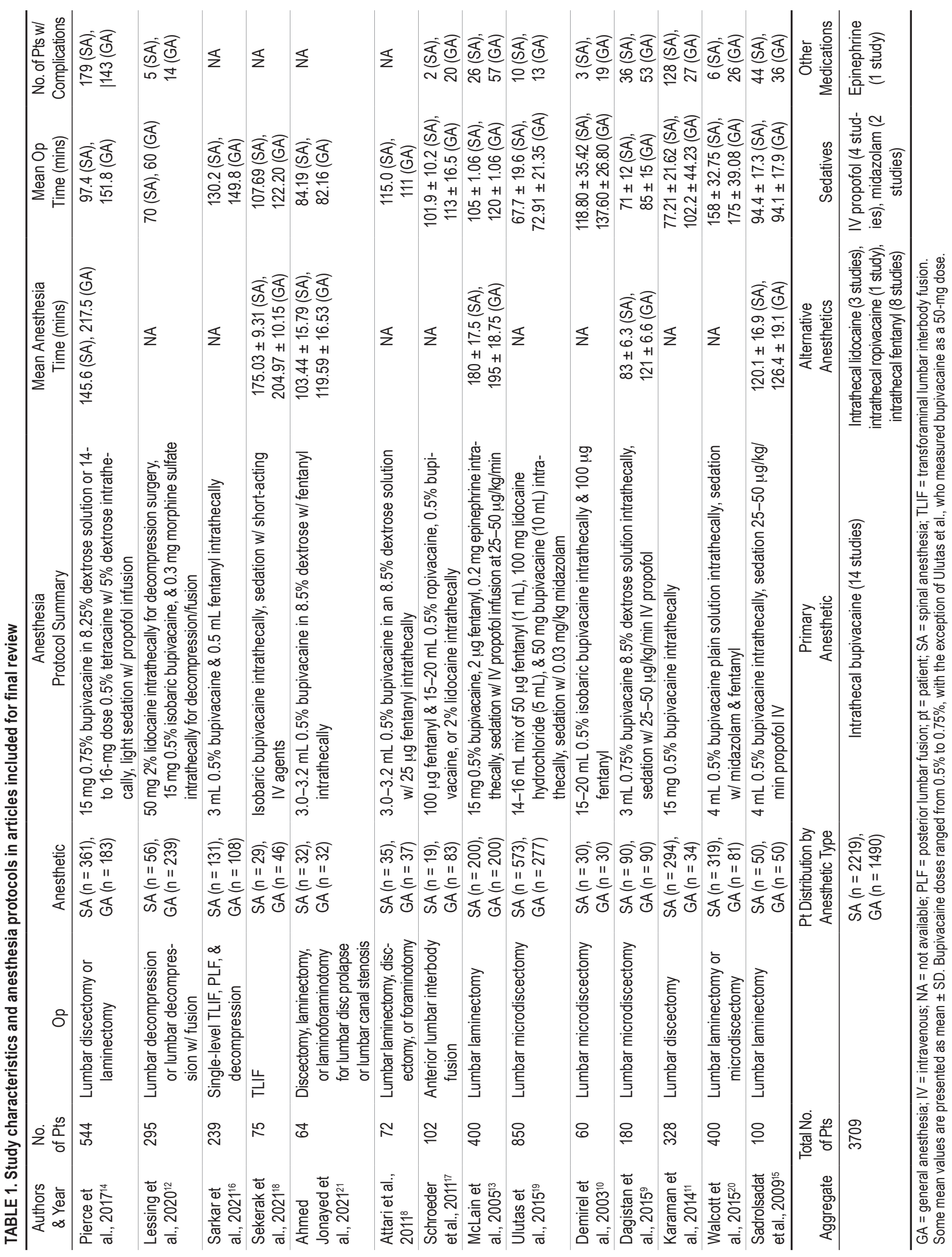




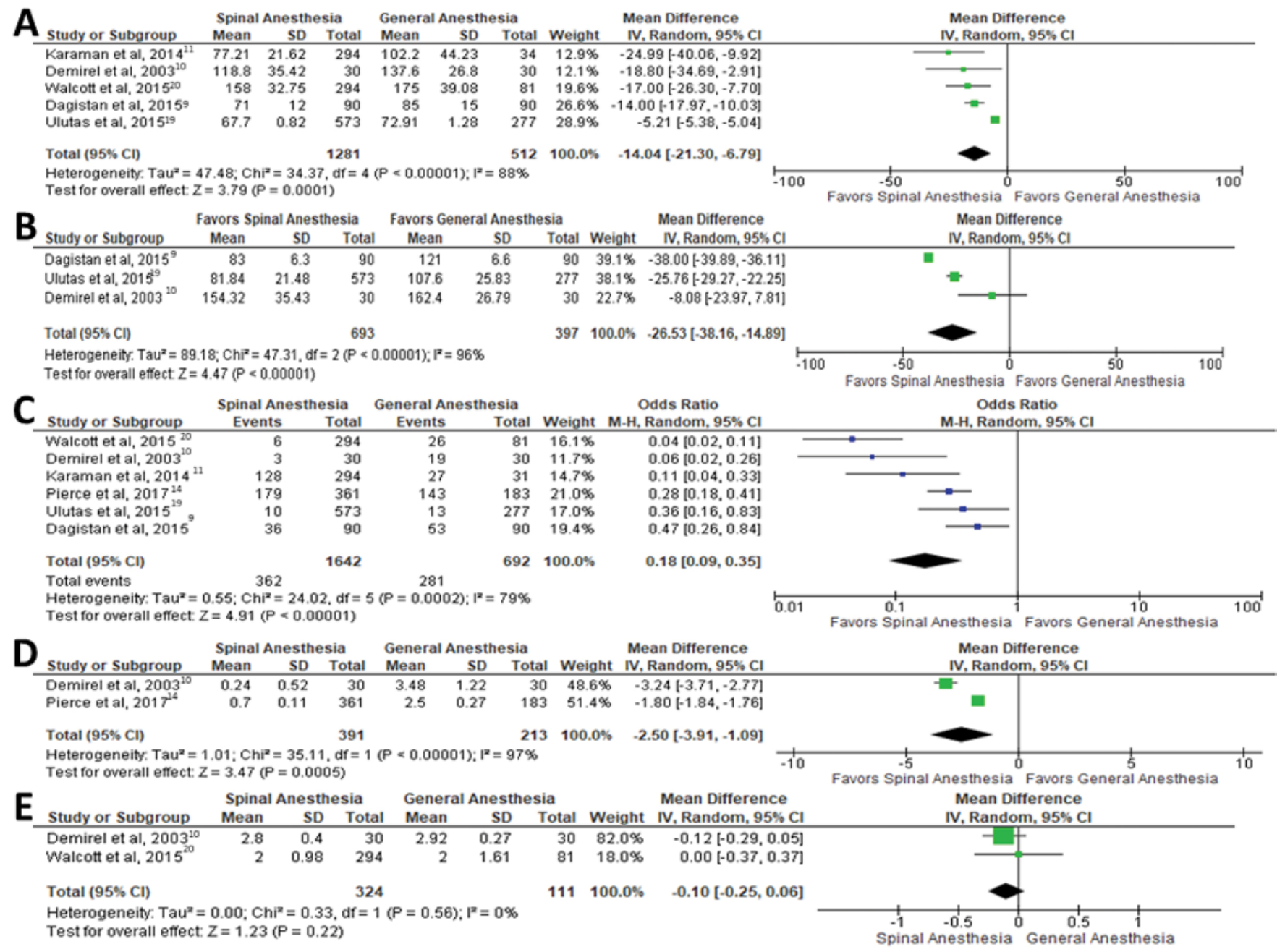

FIG. 3. Pooled analyses of patient outcomes in lumbar discectomies. A: Patients who received spinal anesthesia had significantly shorter mean operating times relative to patients receiving general anesthesia ( $\mathrm{MD}-14.04,95 \% \mathrm{Cl}-21.30$ to $-6.79 ; p=0.0001$ ). B: Patients who underwent spinal anesthesia had significantly shorter total anesthesia times (MD $-26.53,95 \% \mathrm{Cl}-38.16$ to $-14.89 ; p=0.00001)$. C: Patients who received spinal anesthesia were significantly less likely to experience postoperative complications than patients who received general anesthesia (OR 0.18, 95\% Cl 0.09-0.35; $p<0.00001$ ). D: Patients who underwent spinal anesthesia reported lower VAS pain scores postoperatively compared with those who underwent general anesthesia (MD $-2.50,95 \% \mathrm{Cl}-3.91$ to $-1.09 ; p=0.0005)$. E: There were no significant differences in LOS between patients who received spinal anesthesia and those who received general anesthesia (MD $-0.10,95 \% \mathrm{Cl}-0.25$ to $0.06 ; p=0.22$ ).

that improvements in postoperative outcomes may allow spinal anesthesia to be an adequate substitute for general anesthesia in certain lumbar procedures. ${ }^{22}$

Perhaps our most pertinent finding is the consistency with which spinal anesthesia has demonstrated superior outcomes relative to general anesthesia. Our pooled analyses have demonstrated that patients who underwent spinal anesthesia had shorter anesthesia and operative times, with lower risk of postoperative complications and postoperative pain scores compared with patients who underwent general anesthesia. This was evident across all surgical procedures that were evaluated, which ranged from simple lumbar decompression to interbody fusions.

These findings highlight a trend that is only recently gaining traction. Traditionally, spinal anesthesia has been considered a secondary option to general anesthesia in patients with multiple comorbidities and high American So- ciety of Anesthesiologists scores. ${ }^{22}$ However, a landmark paper by Khan et al. in 2014 demonstrated that patients who traditionally were seen as fit for general anesthesia demonstrated equivalent or improved outcomes when receiving spinal anesthesia alone. ${ }^{24}$ All of the above findings may impact and transcend beyond the operating room. Several studies have reported that overall cost can be reduced between $6 \%$ and $10 \%$ with the use of spinal anesthesia. ${ }^{16,18}$ This was confirmed by our meta-analysis in which there was a significant decrease in anesthesia cost in the spinal anesthesia cohort. The reduction in anesthesia and operative times in addition to a decrease in postoperative analgesia use might explain these findings. Garg and colleagues further expanded on their notion of an ideal patient subgroup and determined that patients with normal BMI and a low cardiovascular risk profile may be particularly qualified candidates for awake spine procedures. ${ }^{25}$ 


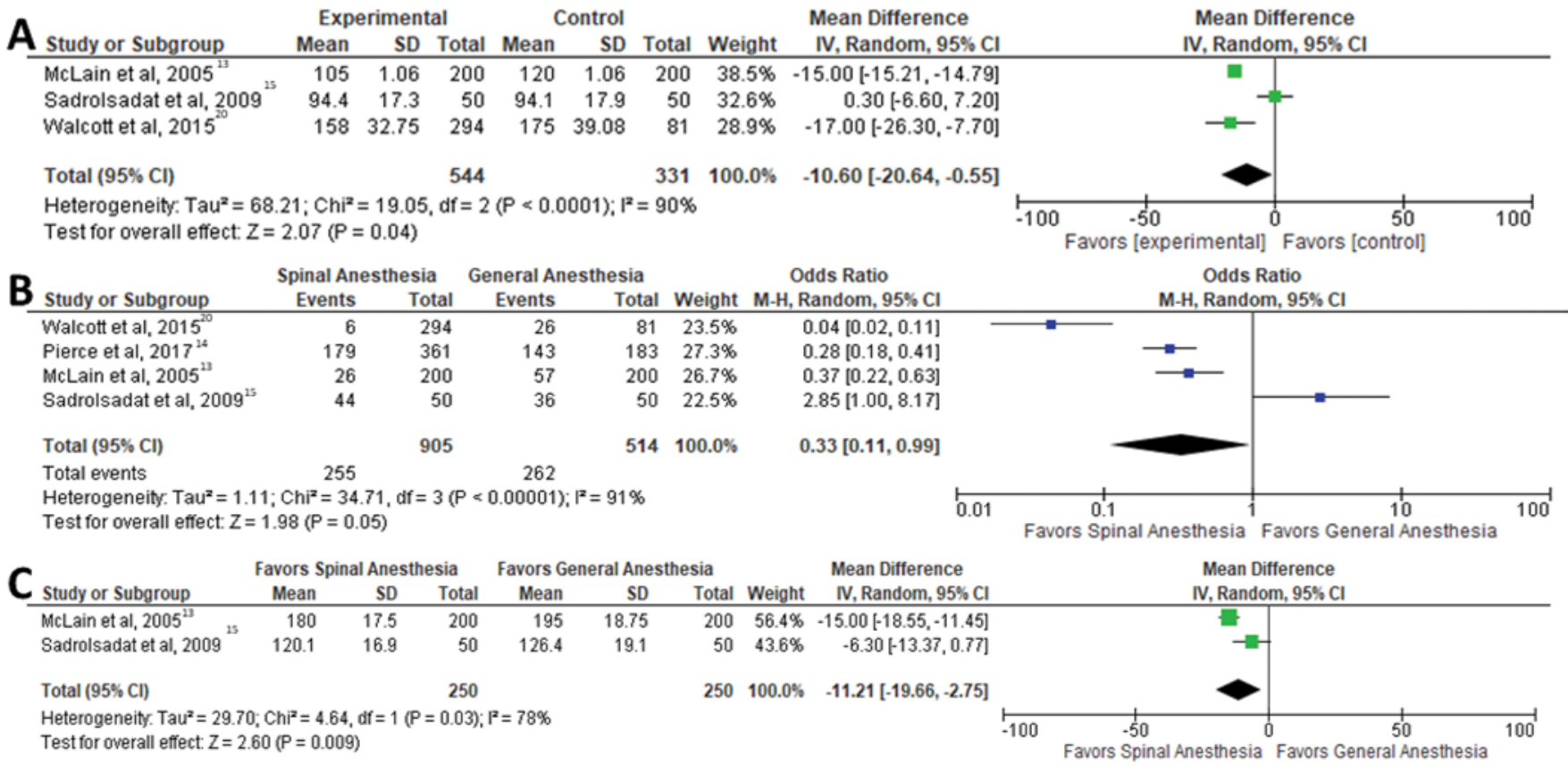

FIG. 4. Analysis of patient outcomes in lumbar laminectomies. A: There were significant differences in total operative time between patients who received spinal anesthesia and patients who received general anesthesia (MD $-10.60,95 \% \mathrm{Cl}-20.64$ to $-0.55 ; p=0.04)$. B: There were statistically significant differences in complication risk (OR $0.33,95 \% \mathrm{Cl} 0.11-0.99 ; p=0.05)$. C: Total anesthesia time was significantly shorter in patients who received spinal anesthesia than in those who received general anesthesia (MD $-11.21,95 \% \mathrm{Cl}-19.66$ to $-2.75 ; \mathrm{p}=0.009$ ).

Although there are some advantages in using spinal anesthesia, there are some clear pitfalls and disadvantages associated with this technique. This method of anesthesia should be reserved for simple and limited lumbar procedures (e.g., minimally invasive lumbar decompression) in which surgical time and expected blood loss are constrained. Nonetheless, recent studies have found that alternative awake protocols are effective and safe in patients undergoing endoscopic lumbar fusions. ${ }^{26}$ Additionally, the local anesthetics utilized in this modality can have variable durations of action which may translate into patient discomfort during the procedure if surgery is prolonged. Also, patients who have high levels of anxiety may not tolerate this alternative. ${ }^{27}$ Furthermore, recent literature has demonstrated that spinal anesthesia may be contraindicated in patients who are at high risk for airway compromise, such as morbidly obese patients or those with chronic obstructive pulmonary disease, likely due to potential difficulties in maintaining airway patency intraoperatively. $22,28,29$

\section{Limitations}

While we performed a meta-analysis of 3709 patients across 14 studies, our primary limitation involved the lack of data on spinal anesthesia in other spinal procedures. The majority of our data was derived from studies investigating the role of spinal anesthesia in lumbar de-

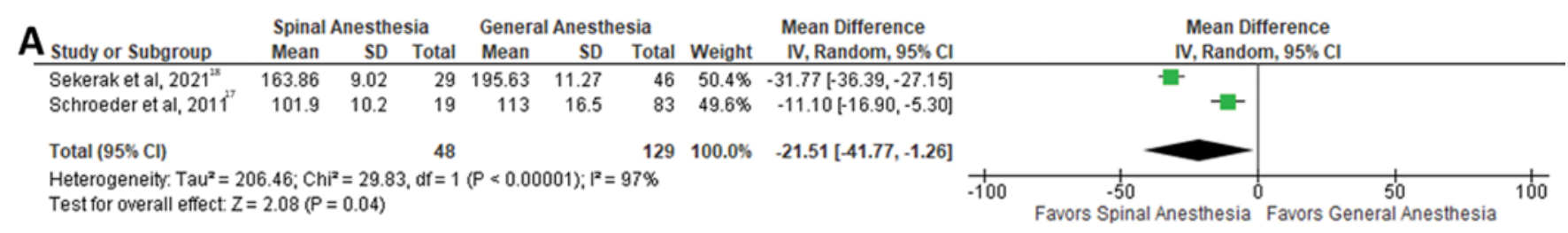

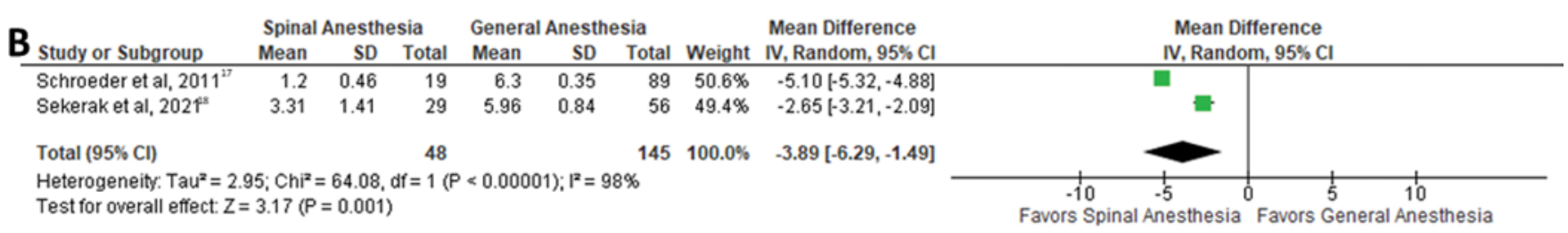

FIG. 5. Analysis of patient outcomes in lumbar fusion. A: Patients who received spinal anesthesia had significantly shorter operative times (MD $-21.51,95 \% \mathrm{Cl}-41.77$ to $-1.26 ; p=0.04$ ). B: Patients who received spinal anesthesia also had significantly lower postoperative pain than patients who received general anesthesia (MD $-3.89,95 \% \mathrm{Cl}-6.29$ to $-1.49 ; \mathrm{p}=0.001$ ). 


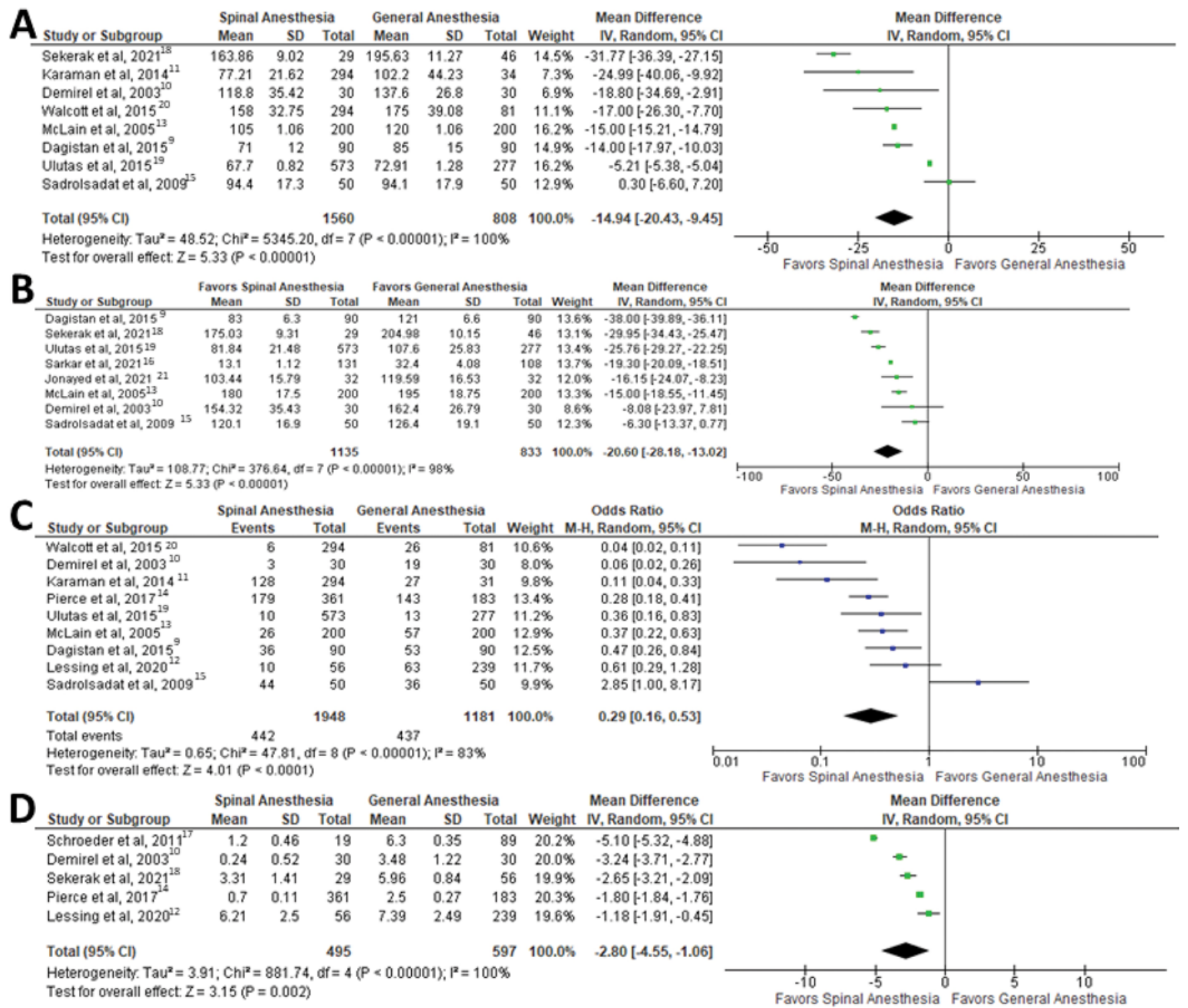

FIG. 6. Analysis of patient outcomes across all investigated surgical techniques. A: Across all studies, patients who received spinal anesthesia had significantly shorter total operative times than those who received general anesthesia (MD -14.94, 95\% $\mathrm{Cl}-20.43$ to $-9.45 ; \mathrm{p}<0.00001$ ). B: Total anesthesia times were significantly shorter for patients who received spinal anesthesia than for those who received general anesthesia (MD $-20.60,95 \% \mathrm{Cl}-28.18$ to $-13.02 ; \mathrm{p}<0.00001)$. C: Complication rates were also significantly lower in patients who received spinal anesthesia compared with patients who received general anesthesia (OR $0.29,95 \% \mathrm{Cl} 0.16-0.53 ; p<0.0001)$. D: Patients who received spinal anesthesia had significantly lower VAS scores than those who received general anesthesia (MD $-2.80,95 \% \mathrm{Cl}-4.55$ to $-1.06 ; p=0.002)$.

compression surgery. Consequently, our results regarding the impact of spinal anesthesia in other lumbar procedures may have been hampered by a lack of studies investigating this topic. Furthermore, all our data were derived from studies investigating the lumbar spine; therefore, we are unable to generalize our findings to procedures performed on other regions of the vertebral column. In addition, it is difficult to control for inherent differences in surgeon preferences in surgical technique and postoperative care as well as discharge protocols. In our review of the literature, we also noted the variability in which several metrics are reported. For example, hospital LOS and postopera- tive analgesic use are reported in various ways, making pooled analyses of these metrics difficult. Thus, there may be further differences between anesthetic techniques that are obscured by a lack of uniform reporting. Finally, many of our articles were written from an anesthetic as opposed to a surgical perspective so that detailed descriptions of surgical techniques are somewhat lacking.

\section{Conclusions}

Our systematic review and meta-analysis has demonstrated certain advantages of awake spinal anesthesia 


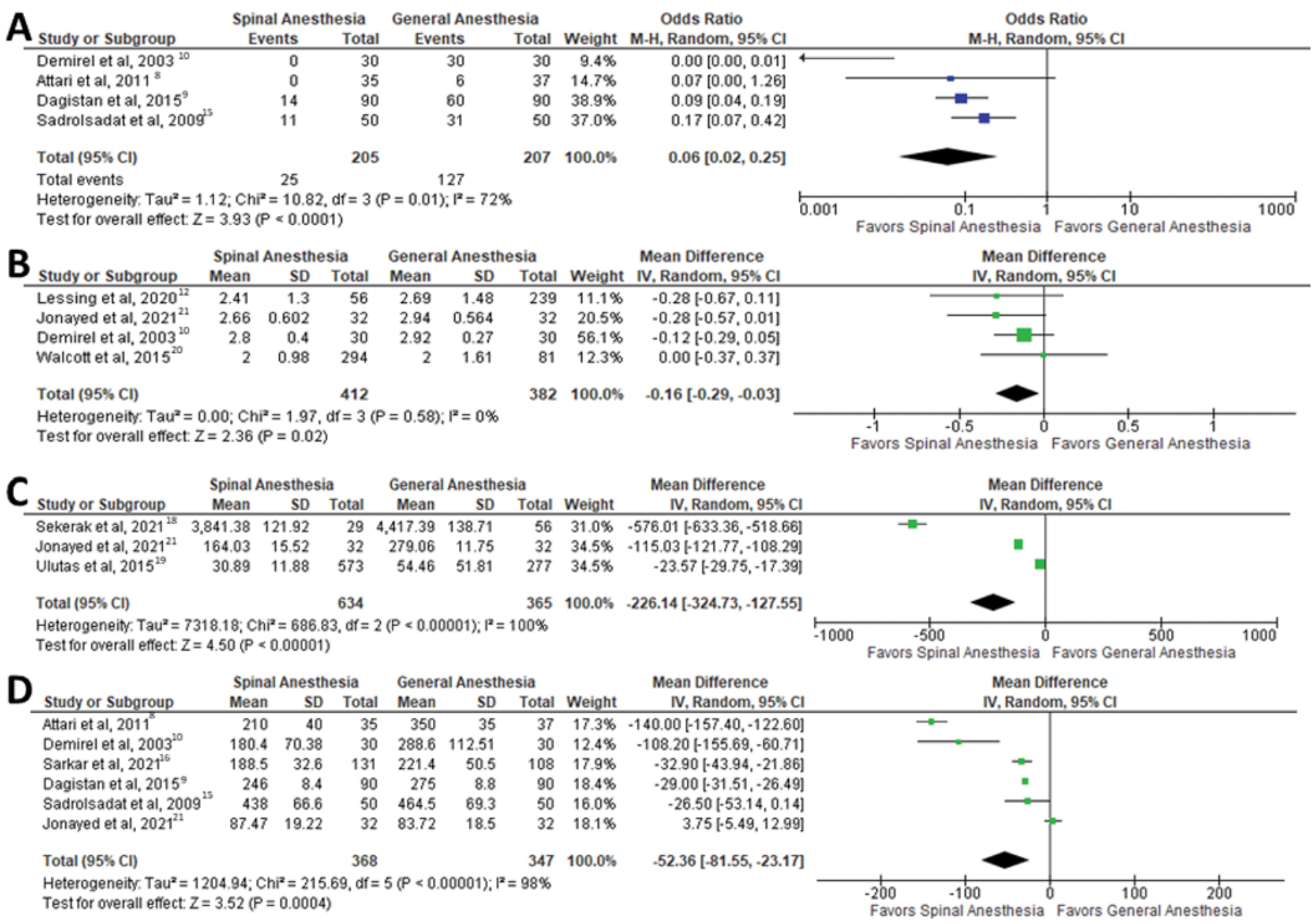

FIG. 7. Global analysis of other postoperative metrics across all studies. A: Patients who received spinal anesthesia were significantly less likely to require postoperative analgesics (OR 0.06, 95\% Cl 0.02-0.25; $p<0.0001$ ). B: Patients who received spinal anesthesia had a significantly shorter total LOS (MD $-0.16,95 \% \mathrm{Cl}-0.29$ to $-0.03 ; p=0.02)$. C: Spinal anesthesia was significantly less costly across all investigated studies (MD $-226.14,95 \% \mathrm{Cl}-324.73$ to $-127.55 ; \mathrm{p}<0.00001)$. D: Patients who received spinal anesthesia had significantly less postoperative blood loss (MD $-52.36,95 \% \mathrm{Cl}-81.55$ to $-23.17 ; p=0.0004$ ).

relative to general anesthesia in multiple metrics in lumbar surgery. We have shown that patients who received spinal anesthesia for awake surgical procedures had significantly shorter operative times and a shorter total time under anesthesia, had decreased postoperative pain, and were significantly less likely to experience postoperative complications. Furthermore, we have shown that these general trends are conserved across several procedures of the lumbar spine. These benefits extend to other metrics as well, including cost, blood loss, and postoperative analgesia use. Studies investigating the utility of spinal anesthesia in a wider range of procedures are necessary to fully explore the value of this anesthetic technique.

\section{References}

1. Kulikov A, Lubnin A. Anesthesia for awake craniotomy. Curr Opin Anaesthesiol. 2018;31(5):506-510.

2. Bajwa SJS, Kulshrestha A. Anaesthesia for laparoscopic surgery: general vs regional anaesthesia. J Minim Access Surg. 2016;12(1):4-9.

3. Sairyo K, Chikawa T, Nagamachi A. State-of-the-art transfo- raminal percutaneous endoscopic lumbar surgery under local anesthesia: discectomy, foraminoplasty, and ventral facetectomy. J Orthop Sci. 2018;23(2):229-236.

4. Basil GW, Wang MY. Trends in outpatient minimally invasive spine surgery. J Spine Surg. 2019;5(1)(suppl 1):S108S114.

5. Meng T, Zhong Z, Meng L. Impact of spinal anaesthesia vs. general anaesthesia on peri-operative outcome in lumbar spine surgery: a systematic review and meta-analysis of randomised, controlled trials. Anaesthesia. 2017;72(3):391-401.

6. De Cassai A, Geraldini F, Boscolo A, Pasin L, Pettenuzzo $\mathrm{T}$, Persona $\mathrm{P}$, et al. General anesthesia compared to spinal anesthesia for patients undergoing lumbar vertebral surgery: a meta-analysis of randomized controlled trials. J Clin Med. 2020;10(1):E102.

7. Zorrilla-Vaca A, Healy RJ, Mirski MA. A comparison of regional versus general anesthesia for lumbar spine surgery: a meta-analysis of randomized studies. J Neurosurg Anesthesiol. 2017;29(4):415-425.

8. Attari MA, Mirhosseini SA, Honarmand A, Safavi MR. Spinal anesthesia versus general anesthesia for elective lumbar spine surgery: a randomized clinical trial. J Res Med Sci. 2011;16(4):524-29. 
9. Dagistan Y, Okmen K, Dagistan E, Guler A, Ozkan N. Lumbar microdiscectomy under spinal and general anesthesia: a comparative study. Turk Neurosurg. 2015;25(5):685-689.

10. Demirel CB, Kalayci M, Ozkocak I, Altunkaya H, Ozer Y, Acikgoz B. A prospective randomized study comparing perioperative outcome variables after epidural or general anesthesia for lumbar disc surgery. J Neurosurg Anesthesiol. 2003;15(3):185-192.

11. Karaman S, Karaman T, Dogru S, Sahin A, Arici S, Tapar H, et al. Retrospective evaluation of anesthesia approaches for lumbar disc surgery. J Anesth Clin Res. 2014;5:4.

12. Lessing NL, Edwards CC II, Dean CL, Waxter OH, Lin C, Curto RA, Brown CH IV. Spinal anesthesia for geriatric lumbar spine surgery: a comparative case series. Int J Spine Surg. 2020;14(5):713-721.

13. McLain RF, Kalfas I, Bell GR, Tetzlaff JE, Yoon HJ, Rana M. Comparison of spinal and general anesthesia in lumbar laminectomy surgery: a case-controlled analysis of 400 patients. J Neurosurg Spine. 2005;2(1):17-22.

14. Pierce JT, Kositratna G, Attiah MA, Kallan MJ, Koenigsberg $\mathrm{R}$, Syre P, et al. Efficiency of spinal anesthesia versus general anesthesia for lumbar spinal surgery: a retrospective analysis of 544 patients. Local Reg Anesth. 2017;10:91-98.

15. Sadrolsadat SH, Mahdavi AR, Moharari RS, Khajavi MR, Khashayar P, Najafi A, Amirjamshidi A. A prospective randomized trial comparing the technique of spinal and general anesthesia for lumbar disk surgery: a study of 100 cases. Surg Neurol. 2009;71(1):60-65.

16. Sarkar S, Banerji A, Chattopadhyaya A, Banerjee S. Lumbar spine instrumented fusion surgery under spinal anaesthesia versus general anaesthesia-A retrospective study of 239 cases. J Clin Orthop Trauma. 2021;18:205-208.

17. Schroeder KM, Zahed C, Andrei AC, Han S, Ford MP, Zdeblick TA. Epidural anesthesia as a novel anesthetic technique for anterior lumbar interbody fusion. J Clin Anesth. 2011; 23(7):521-526.

18. Sekerak R, Mostafa E, Morris MT, Nessim A, Vira A, Sharan A. Comparative outcome analysis of spinal anesthesia versus general anesthesia in lumbar fusion surgery. J Clin Orthop Trauma. 2021;13:122-126.

19. Ulutas M, Secer M, Taskapilioglu O, Karadas S, Aykut Akylimaz A, Baydilek Y, et al. General versus epidural anesthesia for lumbar microdiscectomy. J Clin Neurosci. 2015;22(8): 1309-1313.

20. Walcott BP, Khanna A, Yanamadala V, Coumans JV, Peterfreund RA. Cost analysis of spinal and general anesthesia for the surgical treatment of lumbar spondylosis. J Clin Neurosci. 2015;22(3):539-543.

21. Ahmed Jonayed S, Alam MS, Al Mamun Choudhury A, Akter S, Chakraborty S. Efficacy, safety, and reliability of surgery on the lumbar spine under general versus spinal anesthesia-an analysis of 64 cases. J Clin Orthop Trauma. 2021; $16: 176-181$.
22. Fiani B, Reardon T, Selvage J, Dahan A, El-Farra MH, Endres P, et al. Awake spine surgery: an eye-opening movement. Surg Neurol Int. 2021;12:222.

23. Deng H, Coumans JV, Anderson R, Houle TT, Peterfreund RA. Spinal anesthesia for lumbar spine surgery correlates with fewer total medications and less frequent use of vasoactive agents: a single center experience. PLoS One. 2019;14(6): e0217939.

24. Khan MB, Kumar R, Enam SA. Thoracic and lumbar spinal surgery under local anesthesia for patients with multiple comorbidities: a consecutive case series. Surg Neurol Int. 2014; 5(3)(suppl 3):S62-S65.

25. Garg B, Ahuja K, Sharan AD. Awake spinal fusion. J Clin Orthop Trauma. 2020;11(5):749-752.

26. Chang HK, Huang M, Wu JC, Huang WC, Wang MY. Less opioid consumption with enhanced recovery after surgery transforaminal lumbar interbody fusion (TLIF): a comparison to standard minimally-invasive TLIF. Neurospine. 2020; 17(1):228-236.

27. Mitchell M. Patient anxiety and conscious surgery. J Perioper Pract. 2009;19(6):168-173.

28. Ames WA, Songhurst L, Gullan RW. Local anaesthesia for laminectomy surgery. Br J Neurosurg. 1999;13(6):598-600.

29. Collins LM, Vaghadia H. Regional anesthesia for laparoscopy. Anesthesiol Clin North America. 2001;19(1):43-55.

\section{Disclosures}

Dr. Wang receives royalty payments from DePuy-Synthes Spine, Inc., Children's Hospital of Los Angeles, Springer Publishing, and Quality Medical Publishing; is a consultant for DePuy-Synthes Spine, Inc., Stryker Spine, Medtronic, K2M, and Spineology; is an advisory board member for Vallum; has direct stock ownership in Spinicity, Kinesiometrics, Medical Device Partners, and Innovative Surgical Devices; and has received grants from the Department of Defense.

\section{Author Contributions}

Conception and design: Perez-Roman. Acquisition of data: Govindarajan, Bryant. Analysis and interpretation of data: Govindarajan. Drafting the article: Govindarajan, Bryant. Critically revising the article: Wang, Perez-Roman. Reviewed submitted version of manuscript: all authors. Statistical analysis: Govindarajan, Bryant.

\section{Correspondence}

Michael Y. Wang: University of Miami Miller School of Medicine, Lois Pope Life Center, Miami, FL. mwang2@ med.miami.edu. 